\title{
Design of the MagAO-X Pyramid Wavefront Sensor
}

\author{
Lauren Schatz ${ }^{a}$, Oli Durney ${ }^{b}$, Jared R. Males ${ }^{b}$, Laird Close ${ }^{b}$, Olivier Guyon ${ }^{a b d}$ \\ ${ }^{e}$, Michael Hart ${ }^{a c}$, Jennifer Lumbres ${ }^{a}$, Kelsey Miller ${ }^{a}$, Justin Knight ${ }^{a}$, \\ Alexander Rodack ${ }^{a}$ \\ ${ }^{a}$ University of Arizona, College of Optical Sciences, 1630 E Universtiy Blvd, \\ Tucson, AZ 85719 \\ ${ }^{b}$ University of Arizona, Steward Observatory, Tucson, 933 N Cherry Ave, \\ Tucson, AZ 85721 \\ ${ }^{c}$ Institute for Astronomy, University of Hawaii, 34 Ohia Ku St, Pukalani, HI \\ 96768 \\ ${ }^{d}$ National Astronomical Observatory of Japan, Subaru Telescope, National \\ Institutes of Natural Sciences, Hilo, HI 96720, USA \\ ${ }^{e}$ Astrobiology Center, National Institutes of Natural Sciences, 2-21-1 Osawa, \\ Mitaka, Tokyo, JAPAN
}

\section{ABSTRACT}

Adaptive optics systems correct atmospheric turbulence in real time. Most adaptive optics systems used routinely correct in the near infrared, at wavelengths greater than $1 \mu \mathrm{m}$. MagAO-X is a new extreme adaptive optics (ExAO) instrument that will offer corrections at visible-to-nearIR wavelengths. MagAO-X will achieve Strehls $\geq 70 \%$ at $\mathrm{H} \alpha$ when running the 2048 high-order actuator deformable mirror at $3.7 \mathrm{kHz}$. A visible pyramid wavefront sensor (PWFS) optimized for sensing at 600-1000 $\mathrm{nm}$ wavelengths will provide the high-order wavefront sensing on MagAO$\mathrm{X}$. We present the optical design and predicted performance of the MagAO-X pyramid wavefront sensor. ${ }^{1}$

\section{INTRODUCTION}

MagAO-X is a new visible-to-near-IR extreme adaptive optics system (ExAO) for the $6.5 \mathrm{~m}$ Magellan Clay telescope. Working off of lessons learned from $\mathrm{MagAO}^{3}$ (P.I. Laird Close), and $\mathrm{SCExAO}^{4}$ (P.I. Olivier Guyon), MagAO-X is optimized for high impact science cases, such as a survey of nearby newly formed accreting planets in $\mathrm{H} \alpha .{ }^{1}$ To achieve excellent contrast and resolution MagAO-X will utitlize a 2048 high-order actuator deformable mirror (DM) in conjunction with a cutting edge coronagraph for starlight suppression. A pyramid wavefront sensor will provide high order wavefront sensing. In this paper we present the design and predicted performance of the MagAO-X pyramid wavefront sensor.

The pyramid wavefront sensor (1996 by Ragazzoni $)^{5}$ is a pupil plane wavefront sensor that in effect works as a 2-D Foucault knife-edge test. In commonly used configurations the focal plane is split into four quadrants using a double achromatic prism, (LBTAO, and MagAO), or using 


\begin{tabular}{|l|l|}
\hline Parameter & Requirement \\
\hline Wavelength Range & $600-1000 \mathrm{~nm}$ \\
\hline Pupil Size & 56 pixels; $2.688 \mathrm{~mm}$ \\
\hline Pupil Separation & 60 pixels; $2.880 \mathrm{~mm}$ \\
\hline Pupil Tolerances & $\Delta<1 / 10$ th pixel; $2.4 \mu \mathrm{m}$ \\
\hline Lens Diameter & $10 \mathrm{~mm}<\mathrm{D}<20 \mathrm{~mm}$ \\
\hline
\end{tabular}

Table 1: Parameters for the MagAO-X pyramid wavefront sensor.

two roof prisms, (SCeXAO). Each quadrant of the focal plane is then reimaged, producing four seperate pupil images on the detector. Local wavefront slopes can be calculated using the quadcell centroid of pixel intensities. The number of spatial frequencies measured by the pyramid wavefront sensor is equal to the number of pixels across one of the pupils. For our pyramid wavefront sensor, we use a copy of the double achromatic prism used on LBTAO and MagAO The major design effort is a new camera lens, to image the four pupils onto our OCAM2k detector at the correct size and seperation.

\section{SYSTEM REQUIREMENTS}

The pyramid wavefront sensor of the MagAO-X system consists of a prism pyramid, a camera lens, and a $\mathrm{OCAM}^{2} \mathrm{~K}$ EMCCD detector. We use the same four sided double pyramid as the MagAO and LBTAO systems. The MagAO-X pyramid wavefront sensor is designed to operate from 600-1000nm bandwidth. Figure 1 is the bandpass of the MagAO PWFS. We expect a similar transmission for the MagAO-X PWFS.

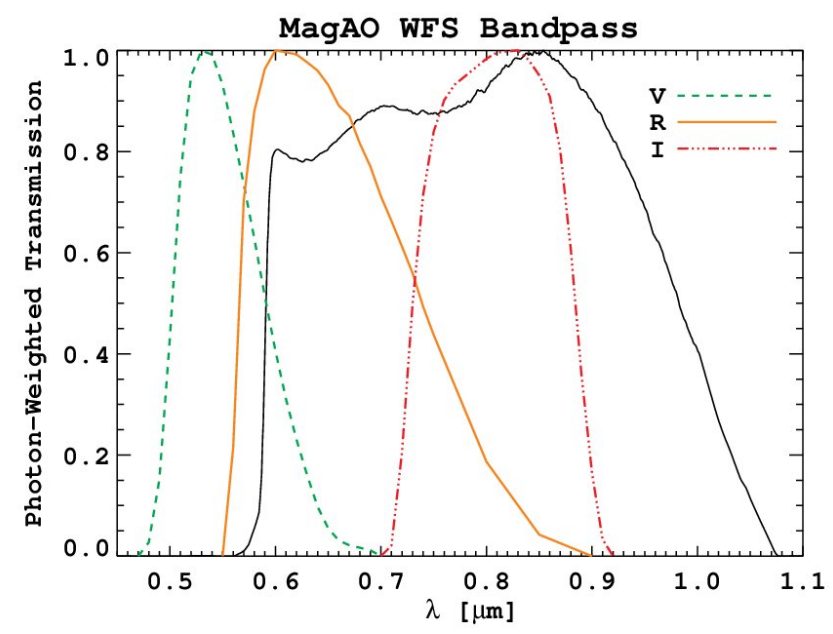

Figure 1: The MagAO pyramid wavefront sensor bandpass, (black curve).

A new camera lens is designed to meet the requirements of the MagAO-X system. These requirements are listed below. The $\mathrm{OCAM}^{2} \mathrm{~K}$ will be used in $2 \mathrm{x} 2$ binning mode, giving us a 48 $\mu \mathrm{m}$ pixel size. 


\subsection{Pyramid Design}

MagAO-X will be using a copy of the pyramid prism used in both the LBTAO and MagAO systems. The pyramid is already in hand. A picture of the pyramid is shown in Figure 2. Details of the design done by Tozzi et. al. are summarized here. ${ }^{2}$ The pyramid used in the WFS is a double pyramid, consisting of two four sided prisms aligned back to back. The total deviation angle needed for the pyramid wavefront sensor is hard to manufacture. Combining two pyramids makes the polishing process easier and at the same time allows us to control chromatic aberrations by using two different glass types. The glass types were chosen using an I.D.L. optimization routine that selected glass combinations from the Shott and Ohara catalog that would give a suitable deflection angle of the double pyramid. The front prism is made from Shott N-SK11, and the back prism is made from Schott N-PSK53.

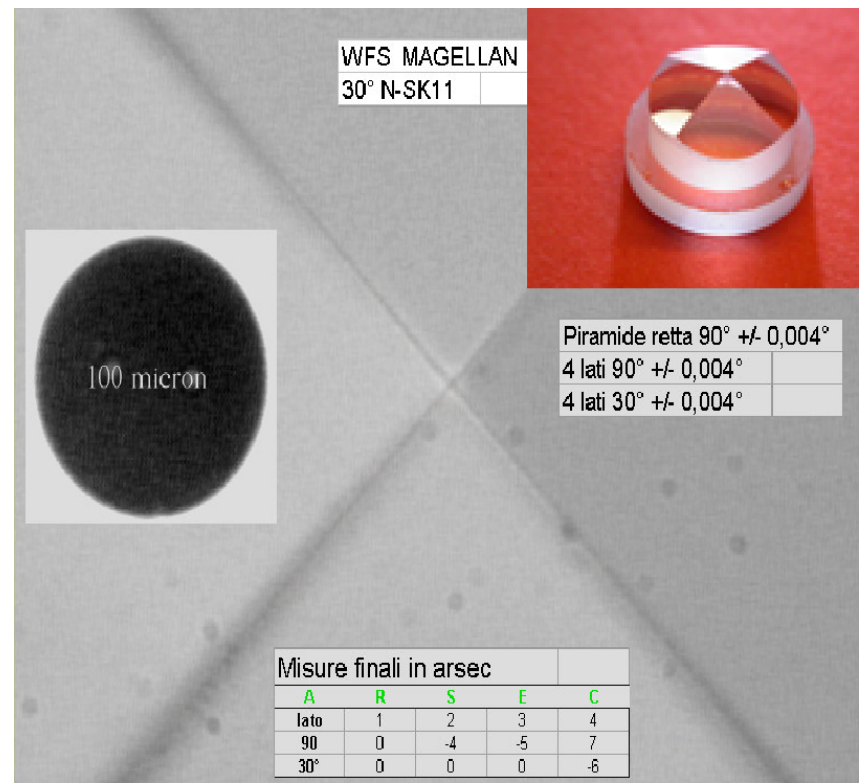

Figure 2: Fabricated pyramid made in Arcetri.

\subsection{Wavefront Sensor Design}

A design of the wavefront sensor was done in Zemax. A table of the element thicknesses (or distances), and radius of curvatures pulled from Zemax is shown in table 3. The wavefront sensor consists of the double pyramid. A F/69 focus created by OAP5\#1 is imaged onto the pyramid tip. A custom achromatic triplet images four pupils onto our $\mathrm{OCAM}^{2} \mathrm{~K}$ wavefront sensor camera. A layout of the wavefront sensor optical path done in both Zemax and SolidWorks is shown in Figure 4. Not shown upstream is the wavefront sensor dichroic pickoff. Except for the high precision flat and dichroic, the light to the wavefront sensor uses the same optical surfaces as the rest of the upstream system to reduce non common path errors. The double pyramid was modeled by the Arcetri team in Zemax, and that same model is used here. A custom achromatic triplet was designed to give the correct pupil size and distances. The two windows in the $\mathrm{OCAM}^{2} \mathrm{~K}$ detector are included in the design for completeness. The expected pupil footprint on the image plane for $800 \mathrm{~nm}$ wavelength is given in Figure 3. 


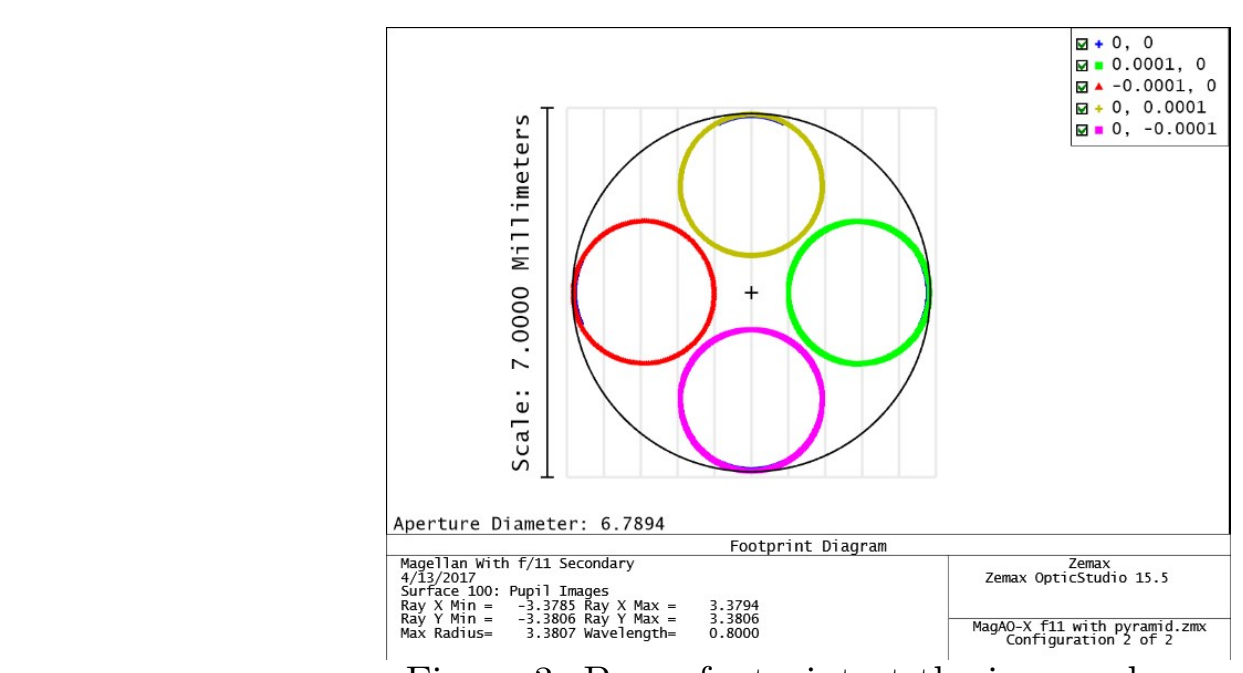

Figure 3: Beam footprint at the image plane.

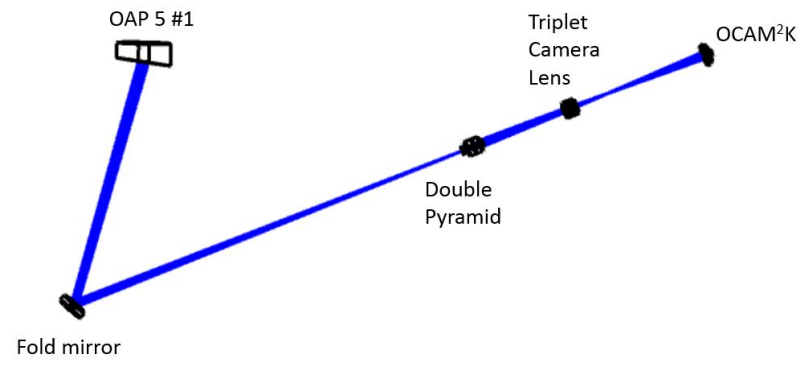

(a) Optical path in Zemax.

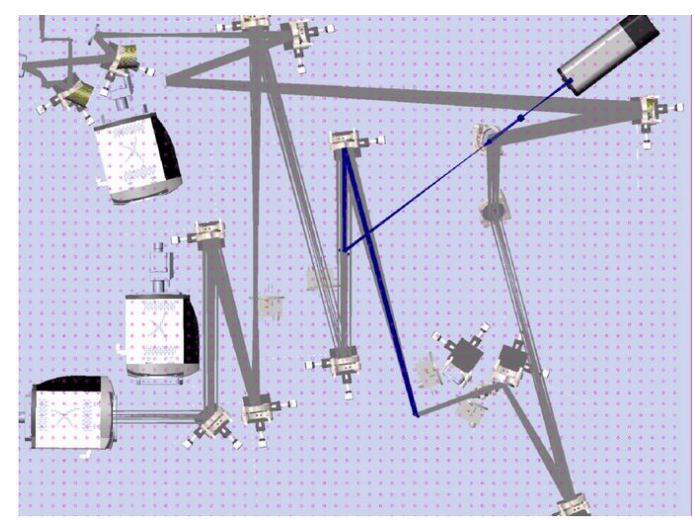

(b) Optical path in SolidWorks

Figure 4: Optical path of the pyramid wavefront sensor. The Zemax ray trace was imported into SolidWorks for the optomechanical design.

\subsection{Achromatic Triplet Design}

Pupil sizes and distances are a vital parameter in the operation and performance of pyramid wavefront sensors. A custom achromatic triplet was designed in Zemax. A schematic of the lens is shown in Figure 5. The OPD error expected from the triplet is expected to be less than 0.5 waves across our wavelength band. The OPD fan is given in Figure 6.

A tolerance analysis was preformed to determine lens performance as a function of wavelength and manufacturing constraints. The tolerancing was done using parameters from the Precision grade Optimax manufacturing tolerancing chart. Reasonable values of alignment errors were estimated and included in the tolerancing analysis. The figure of merit used was the RMS angular radius of the lens because the pyramid is an afocal system. A 500 trial Monte Carlo simulation was done for three wavelengths, $600 \mathrm{~nm}, 800 \mathrm{~nm}$, and $1000 \mathrm{~nm}$. At each wavelength the nominal, mean, and worst RMS angular size (twice the angular radius) was recorded. The difference of the mean and worst angles with respect to the nominal value was calculated. That change in angle was propagated through the system to estimate the change in size we would 


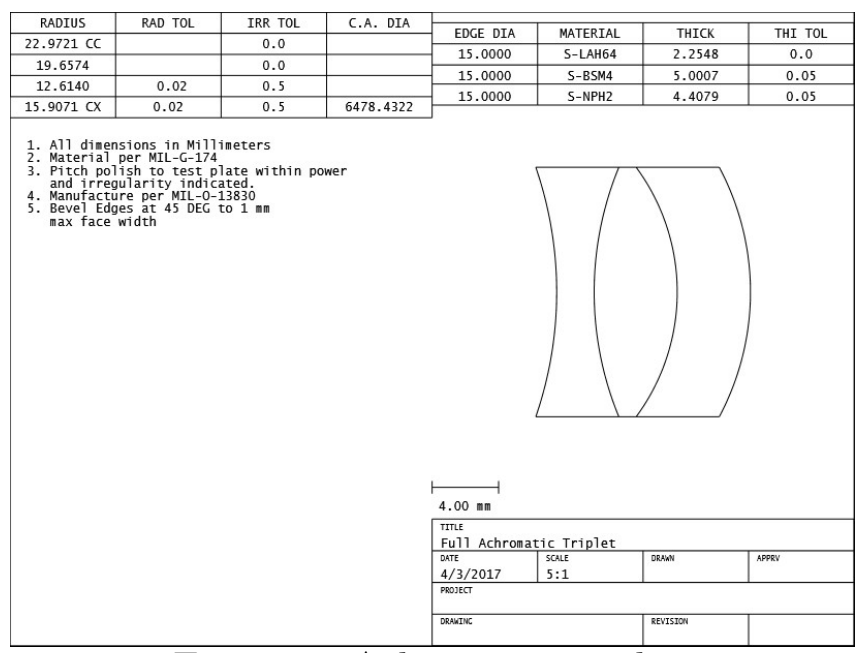

Figure 5: Achromatic triplet.

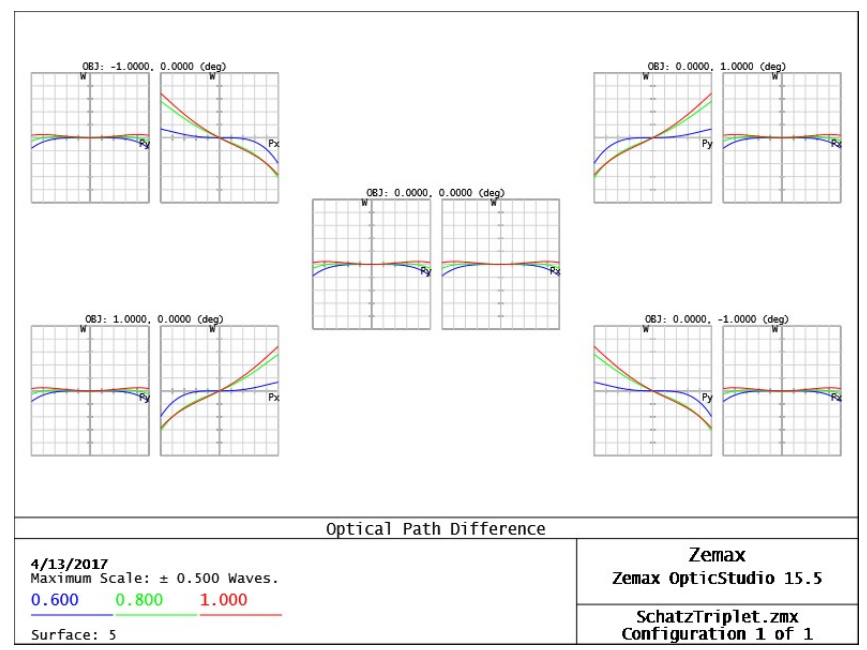

Figure 6: OPD fan of the achromatic triplet.

expect. The propagation is shown in Figure 7 , where $\theta_{n}$ is the nominal RMS angular size, and $\theta_{\Delta}$ is the change in RMS angular size we use to calculate the estimated change $\Delta y$. The distances $x_{1} \ldots x_{5}$ were taken from the Zemax design, and the indexes $n_{1}, n_{2}, n_{3}$ correspond to air, BK-7, and Sapphire respectively. The index of refraction was adjusted for the different wavelengths when the propagation was calculated. The propagation was calculated using trigonometry and Snell's law. The results are summarized in Figure 8, where the change in size in nanometers is graphed against wavelength. At worst we expect about a $45 \mathrm{~nm}$ change in pupil size and separation, and no change on average. Both are well within our tolerance of the change being no greater than $1 / 10$ th a pixel, or $2.4 \mu \mathrm{m}$.

\section{SYSTEM PERFORMANCE}

A simulation of the expected partial illumination of pupil pixels was done in MATLAB. A binary model of the MagAO-X pupil was generated with 10 times the spatial sampling than our expected PWFS pupil. We then bin down to the expected pupil sampling of our PWFS. That is 


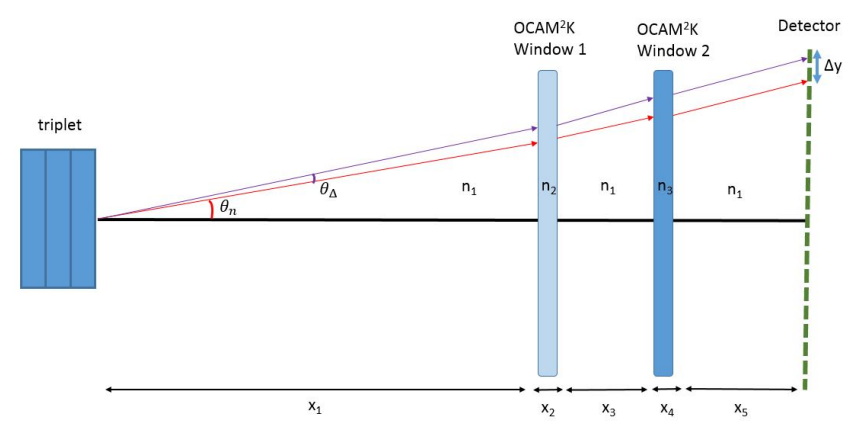

Figure 7: Diagram of the light propagation path used to calculate the change in pupil size.

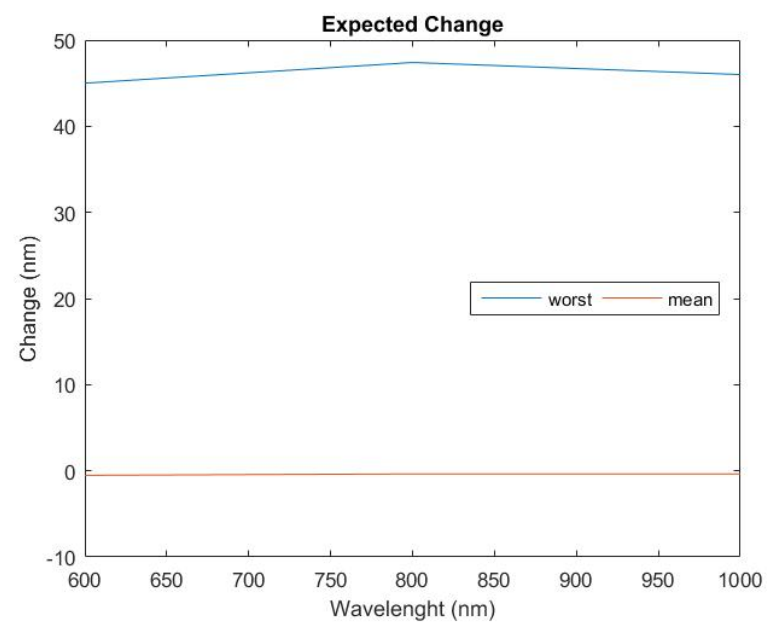

Figure 8: Expected change in pupil size as a function of wavelength.

we start with a pupil of 560 by 560 pixels, and bin down to a 56 by 56 pixel pupil by summing 10 by 10 pixel bins and normalizing. The expected illumination pattern is shown in Figure 9.a. A table of the pixel counts are given in Table 2. where the pixel value is on the X-axis, and the number of pixels with that value are given on the Y-axis. We expect 1958 fully illuminated pixels within our pupil.

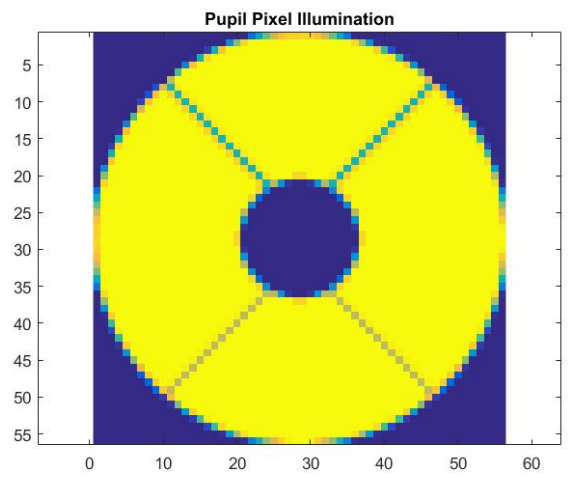

Figure 9: Expected pupil illumination on the PWFS. 


\begin{tabular}{|l|l|}
\hline \% Illumination & \# of Actuators \\
\hline $100 \%$ & 1958 \\
\hline $90 \%$ & 166 \\
\hline $80 \%$ & 24 \\
\hline $0 \%$ & 46 \\
\hline $60 \%$ & 20 \\
\hline $50 \%$ & 18 \\
\hline$<50 \%$ & 904 \\
\hline
\end{tabular}

Table 2: Pixel illuminations in the 56 by 56 pixel pupil. 


\begin{tabular}{|l|l|l|l|}
\hline Element \# & Surface & Radius of Curvature $(\mathrm{mm})$ & Thickness $(\mathrm{mm})$ \\
\hline 76 & OAP5\#1 & 1220.84 & 0 \\
\hline 77 & Propagation \#1 & Infinity & 200 \\
\hline 78 & Coordinate Break & Infinity & 0 \\
\hline 79 & Fold Mirror 2 & Infinity & 0 \\
\hline 80 & Coordinate Break & Infinity & -389.63 \\
\hline 81 & Int. Focal Plane (f/69) & Infinity & 0 \\
\hline 82 & dummy Pyramid Entrance & infinity & -1.5 \\
\hline 83 & PYR 30 & Non-Sequential & 0 \\
\hline 84 & Dummy Out & Non-Sequential & 0 \\
\hline 85 & PYR 30॰ Base & Non-Sequential & -6.16 \\
\hline 86 & PYR 28o base & Non-Sequential & -6.23 \\
\hline 87 & Dummy In & Non-Sequential & 0 \\
\hline 88 & PYR 28० & Non-Sequential & 0 \\
\hline 89 & Dummy Out & Non-Sequential & 0 \\
\hline 90 & Propagation & Infinity & -80 \\
\hline 91 & Triplet Front Surface & -15.88 & -4.41 \\
\hline 92 & Triplet & -12.612 & -5.00 \\
\hline 93 & Triplet & 19.13 & -2.21 \\
\hline 94 & Triplet Back Surface & -23.04 & 0 \\
\hline 95 & propagation & Infinity & -130 \\
\hline 96 & OCAM window & Infinity & -3 \\
\hline 97 & propagation & Infinity & -0.82 \\
\hline 98 & CCD 220 OCAM window & Infinity & -0.9 \\
\hline 99 & Focal Plane & Infinity & -2.83 \\
\hline & & & \\
\hline
\end{tabular}

Table 3: Table of Zemax surface elements 


\section{CONCLUSIONS AND FUTURE WORK}

The MagAO-X pyramid wavefront sensor has been optimized for wavefront sensing from 600 to $1000 \mathrm{~nm}$. A custom acromatic triplet was designed in Zemax to give the appropriate pupil seperation (60 pixels center to center) and sampling (56 pixels across the pupil). We expect to control a maximum of 1958 modes on our 2048 actuator deformable mirror. Future work will see the pyramid wavefront sensor comissioned. In parallel we will be working with the University of Arizona Extreme Adaptive Optics and Coronagraph test bed to study the performance of different pyramid archetectures and reconstruction matrices.

\section{REFERENCES}

1. J. R. Males, MagAO-X team, "MagAO-X Preliminary Design Review", Technical document. (April 24, 2017)

2. A. Tozzi, P. Stefanini, E. Pinna, S. Esposito, "The Double Pyramid wavefront sensor for LBT", in Adaptive Optics Systems, Proceedings of SPIE, (2008). Vol. 7015, 701558

3. K. M. Morzinski, L. M. Close, J. R. Males, D. Kopon, P. M. Hinz, S. Esposito, A. Riccardi, A. Puglisi, E. Pinna, R. Briguglio, M. Xompero, F. Quirs-Pacheco, V. Bailey, K. B. Follette, T. J. Rodigas, Y. Wu, C. Arcidiacono, J. Argomedo, L. Busoni, T. Hare, A. Uomoto, A. Weinberger, "MagAO: Status and on-sky performance of the Magellan adaptive optics system", Proc. SPIE 9148, Adaptive Optics Systems IV, 914804 (21 July 2014); doi: 10.1117/12.2057048; http://dx.doi.org/10.1117/12.2057048

4. T. Currie, O. Guyon, F. Martinache, C. Clergeon, M. McElwain, C. Thalmann, N. Jovanovic, G. Singh, T. Kudo, "The Subaru Coronagraphic Extreme Adaptive Optics Imager: First Results and On-Sky Performace". Proc. IAU 299/Victoria conference proceedings. (15 July 2013); arXiv:1307.4093

5. R. Ragazzoni, "Pupil plane wavefront sensing with an oscillation prism", Journal of Modern Optics. (30 May 1995); http://dx.doi.org/10.1080/09500349608232742 